\title{
Palpation Instrument for Augmented Minimally Invasive Surgery
}

\author{
Maria Vatshaug Ottermo \\ Department of Engineering \\ Cybernetics, Norwegian \\ Trondheim, Norway \\ Email: ottermo@itk.ntnu.no
}

Cybernetics, Norwegian

Øyvind Stavdahl

Department of Engineering

University of Science and Technology University of Science and Technology

\author{
Trondheim, Norway \\ Email: Oyvind.Stavdahl@sintef.no
}

\author{
Tor A. Johansen \\ Department of Engineering \\ Cybernetics, Norwegian
}

University of Science and Technology

Trondheim, Norway

Email: torj@itk.ntnu.no

\begin{abstract}
A preliminary design of a remote palpation instrument for Minimally Invasive Surgery (MIS) is given. The lack of the tactile sense in MIS limits the surgeon's abilities to examine and palpate internal organs. Based on this problem, the aim of this paper is to describe a surgical instrument which will serve as an extension of the surgeon's fingers. A piezoelectric sensor array attached to the instrument's end effector will provide tactile information, which will be sent to the surgeon's fingers via a tactile display to provide a feeling of the shape and hardness of the tissue. The sensor array is $24 \mathrm{~mm} * 8 \mathrm{~mm}$ and consists of 30 piezoelectric sensors, while the tactile display constitutes of $\mathbf{3 0}$ micro motors adding up to a total size of $32 \mathrm{~mm} * 18 \mathrm{~mm} * 45 \mathrm{~mm}$.
\end{abstract}

\section{INTRODUCTION}

Minimally Invasive Surgery (MIS) is one of the methods that makes use of new and complex technology to perform surgery. In MIS, the operation is performed with instruments and viewing equipment inserted into the body through small incisions created by the surgeon. Although this method has many advantages, including minimization of surgical trauma and damage to healthy tissue, it also has disadvantages due to reduced dexterity, workspace and sensory input to the surgeon, which is only available through a single video image. The lack of sensory input through the tactile sense is maybe the most important disadvantage using MIS, as palpation is a powerful tool in locating hidden anatomical structures and evaluating tissue properties. A good example is detection of tumors. Since they tend to be harder than the surrounding tissue, tactile feedback can indicate the presence, size and exact location of a tumor, thereby enhancing the chances of performing successful surgery. Therefore we propose an instrument containing a tactile sensor and a tactile actuator to help overcome this problem (see figure 1).

A tactile sensor measures the spatially distributed parameters of contact on the surface between the sensor and an object, including touch, vibration, pressure and tickle senses, and therefore a tactile sensing array can be considered to be a coordinated group of touch sensors [1].

A tactile display on the other hand is a device that stimulates the skin to generate a sensation of contact. The skin responds to several distributed physical quantities; the most important are perhaps high-frequency vibrations,

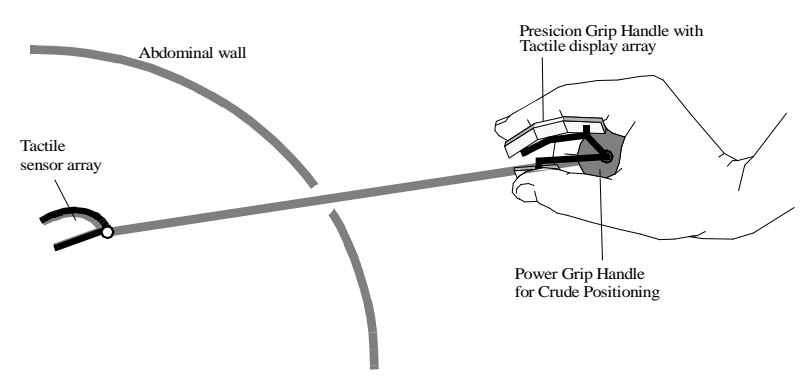

Fig. 1. A sketch of the instrument with tactile sensor and display

small-scale shape or pressure distribution, and thermal properties [2].

Although not necessarily designed for use with instruments for Minimally Invasive Surgery, many tactile sensors and displays have been made. We will only consider a few of the most important designs. A tactile sensor array is considered to be easier to design than a tactile display. It is also the most mature technology and includes work based on capacitive [4], piezoelectric [1], mechanical [5], resistive [6] and magnetoresistive [7] techniques. Most tactile displays use an array of stimulators in contact with the skin to stimulate mechanoreceptors in the finger tip. Previous designs include use of shape memory alloy [3], pneumatics [8], voice coils [9], piezoelectricity [10] and servomotors [11]. However, due to tradeoffs between display bandwidth and actuator density, and also size and weight of the system, no completely satisfactory solution has emerged.

The idea of remote palpation instruments is not new. William Jeane Peine at Harvard University designed a prototype which was able to locate lumps in rubber models [3]. On this instrument the sensor array was based on capacity [4]. It had limitations due to lack of sensitivity, the number of rows in the array which was restricted to one, and stress concentrations induced by edge effects. The tactile shape display was built on shape memory alloy technology and was restricted by the configuration of the pins (a single row), fidelity in pin motion control and size and weight of the device [3]. 
The limitation on number of rows in the previous design by Peine [3] will be addressed for both the sensor and the actuator in the design described in this paper, as will the the size and weight of the display. We propose a sensor array that consists of $302 * 2 * 0.5 \mathrm{~mm}^{3}$ piezoelectric sensor elements in a 3-by-10 pattern, which including spacing adds up to a total size of $24 * 8 \mathrm{~mm}^{2}$. It is designed to measure contact forces between the array and some internal tissue of the body. However, there are other potential application areas for such a sensor, its main advantages being that it is quite small and inexpensive which makes it suitable for applications that require both discrimination of many signals in a small area and disposability. The proposed display for our application consists of 30 micro DC brushless, sensorless motors in an array. The aim of the design is to allow for testing of not only normal, but also tangential forces in the display. The main advantage with our display compared to others will be its small size and high resolution of the actuator pins.

\section{TACtile Sensor ArRay}

\section{A. Principle}

The sensor array is based on piezoelectricity, which is the ability of certain crystals to produce a voltage when subjected to mechanical stress. Size and spacing of the elements were chosen with reference to theories on twopoint discrimination, which on the human finger tip is around 1-2 mm, see eg. [12]. The shape and size of the array were chosen to resemble that of the human finger tip. A thin protective layer of silicone rubber is adhered to the surface of the sensor to provide low pass filtering [13] and enhance mechanical robustness.

For this sensor, the Pz27 material was used (Ferroperm Piezoceramics A/S). Pz27 is an all-round soft PZT material suitable for medical instrumentations because of its high coupling factors, high charge coefficients, high Curie temperature, low mechanical quality factor and low temperature coefficients.

\section{B. Design}

As the forces exerted to the sensor array will be large, typically around 5 to $15 \mathrm{~N}$, the requirements for mechanical stability are high. A series of conductive adhesives were tested to find a satisfactory attachment process. However, all the adhesives were considered unsatisfactory for the purpose, so soldering was found to be the best way to mount all different layers of the sensor array. Since all the layers can not be soldered simultaneously, solders with different melting points are needed. First, the elements are soldered to their dedicated spots on a flexible circuit board with a regular solder, being cautious not to exceed the Curie Temperature. The board with three sensor elements mounted is shown in figure 2 .

Second, a copper plate which serves as a common ground for the piezoelectric elements is soldered to an Olympus $10 \mathrm{~mm}$ Laparoscopic Grasper with a solder designed for steel/aluminum surfaces $\left(215^{\circ} \mathrm{C}\right.$ melting point $)$. Third, the array of piezoelectric elements is attached to the

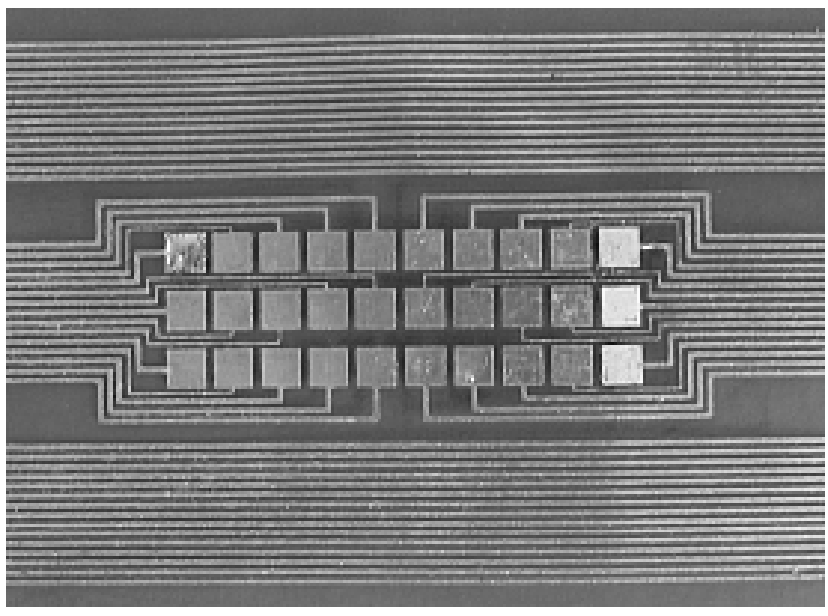

Fig. 2. The flexible circuit board with three sensors mounted

common copper ground using a special solder with melting point at $96^{\circ} \mathrm{C}$. In addition a silicon rubber is attached to the sensor surface to serve as a spatial low pass filter between the elements. It will also protect the elements and board from dirt and make it more mechanically stable.

The circuit board is connected to an amplifier circuit, which in turn is connected to a dSpace DS2002 Multichannel ADC board (dSPACE GmbH). The signals are read from the ADC board using dSpace software (mlib library) and Matlab's Simulink toolbox (The MathWorks, Inc).

\section{TACTILE Display}

\section{A. Principle}

Use of motors in a tactile display has been tested earlier showing good performance, but it has been limited by the size of the display [11]. The mentioned display consisted of $36 \mathrm{RC}$ Servomotors and had a height of $11.9 \mathrm{~cm}$ and a length of $7.6 \mathrm{~cm}$. For our display the total size will be in the range of only one of these servomotors. The motors we use are of type SLB-06H1PG79 from Namiki Precision Jewels (Namiki Precision Jewels CO.LTD) with a gearhead reduction ratio of 1:79, and measure maximum $2.4 \mathrm{~mm}$ in diameter and $12 \mathrm{~mm}$ in length (including shaft), see figure 3 and 4 .

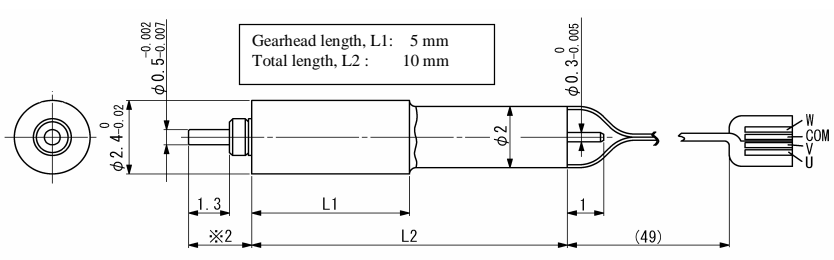

Fig. 3. A sketch of the motor (courtesy of Namiki Precision Jewels CO.LTD)

As mentioned earlier the proposed display will consist of 30 motors adding up to a total size of $32 * 18 * 45 \mathrm{~mm}^{3}$. For the first version which will only be able to indent the finger vertically, the principle is to attach a tiny screw to 


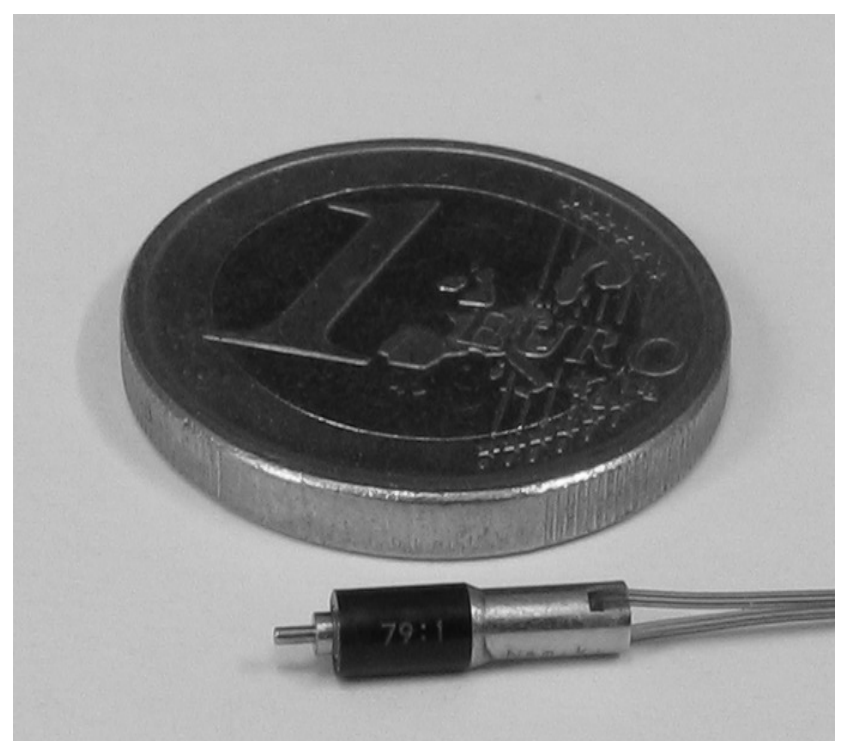

Fig. 4. Motor

the gearhead shaft which screws a top up and down when the shaft rotates, see figure 5 .

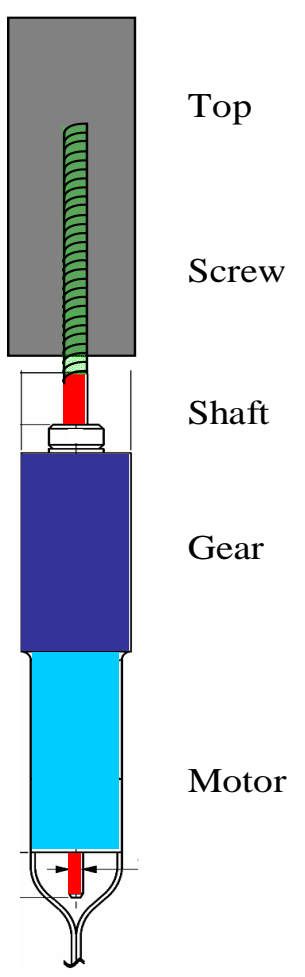

Fig. 5. Integration of tactor and motor

The height of each tactel (TACTile ELement) is determined by an electric signal and will be a function of the force exerted on the tactile sensor elements. With this design, the top of the tactel can easily be replaced, allowing for testing with different shapes, heights and sizes. According to Moy, Wagner and Fearing [8] the force required is estimated to $1 \mathrm{~N}$ per tactor when the actuator density is 1 per $\mathrm{mm}^{2}$, with up to $2 \mathrm{~mm}$ indentation and a bandwidth $>50 \mathrm{~Hz}$ in an ideal tactile display. However, studies have also shown that typical total palpation forces usually range between 5 and $15 \mathrm{~N}$, where $15 \mathrm{~N}$ is a realistic limit for palpation in vivo [3]. Therefore we decided to use a mean palpation force of $10 \mathrm{~N}$ as a measure of required force, meaning that each of the 30 motors had to be capable of producing $0.3 \mathrm{~N}$. The motors are available with different gearhead reductions; 1:4.3-18-79-337, and testing was done to find the most suitable motor. They were tested with a screw with diameter of $1.4 \mathrm{~mm}$, length of $7 \mathrm{~mm}$ and raise of $0.3 \mathrm{~mm} /$ round. The experiment showed that the motor with gearhead reduction ratio of 1:79 had a maximum velocity of $7.24 \mathrm{~mm} / \mathrm{s}$. It could also lift a load of $97 \mathrm{~g}$ (approximately $0.95 \mathrm{~N}$ ) at a velocity of $1.85 \mathrm{~mm} / \mathrm{s}$.

\section{B. Design}

The shape of the display is shown in figure 6 .

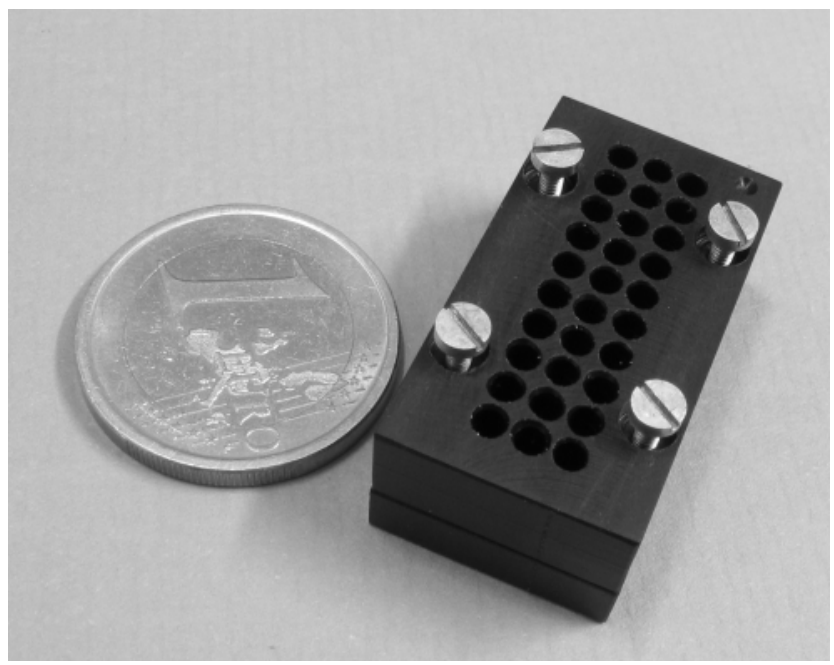

Fig. 6. Display housing without tactels

Each hole in the display housing shown in figure 6 corresponds to a cylinder that is designed to house one motor. One of the challenges of designing the display has been to make it mechanically stable without damaging the gears which are encapsulated in plastic. Another problem is that the motor is only loosely attached to the gear. Therefore the cylinders' inner design has varying diameter to make sure the gear and motor will not detach without putting any pressure on the gearhead (see figure 7).

A sheet of silicone rubber between the two layers also aids in stabilizing the design (see figure 8).

\section{Drive Circuit}

The drive circuit designed to operate the motors is a SSD04 3-phase sensorless drive circuit from Namiki and is shown in figure 9 .

Since we need 30 of these drive circuits it will be too big. Therefore a control strategy for the motors with a design that integrates the 30 driver circuits in one chip (FPGA) is 


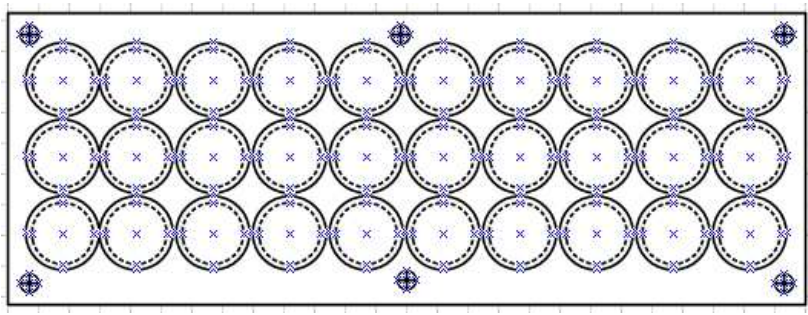

Fig. 7. Cylinders inner design, dotted lines indicating a smaller diameter

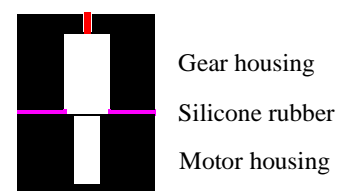

Fig. 8. A sketch of one motor housing with silicone rubber sheet (side view)

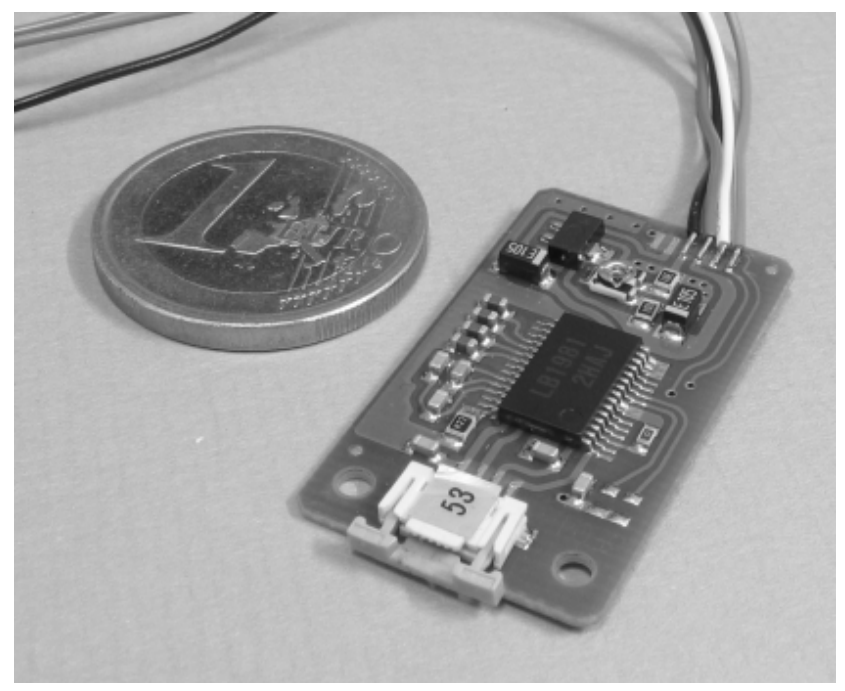

Fig. 9. The SSD04 driver circuit

currently being designed. This circuit will control all the motors in parallel. Another disadvantage with the SSD04 drive circuit it that it uses two phases to control the motor and one to sense each motor revolution via induced voltage in the third phase. With our circuit we will commutate the motor in a stepper motor fashion, allowing for all phases to be used for control.

\section{FUTURE WORK}

The design of a remote palpation instrument to be used in Minimally Invasive Surgery has been described. Problems with earlier prototypes that are addressed include the number of of rows in both the tactile sensor and actuator, and the size and weight of the display.

Future work for the sensor will include a psychophysical experiment aiming at testing the performance of the sensor and exploring the need for tactile feedback in palpation.
In this experiment dSpace hardware and software will be used for sampling of the data, while Matlab will be used for presentation. The data will typically be presented as shown in figure 10 .

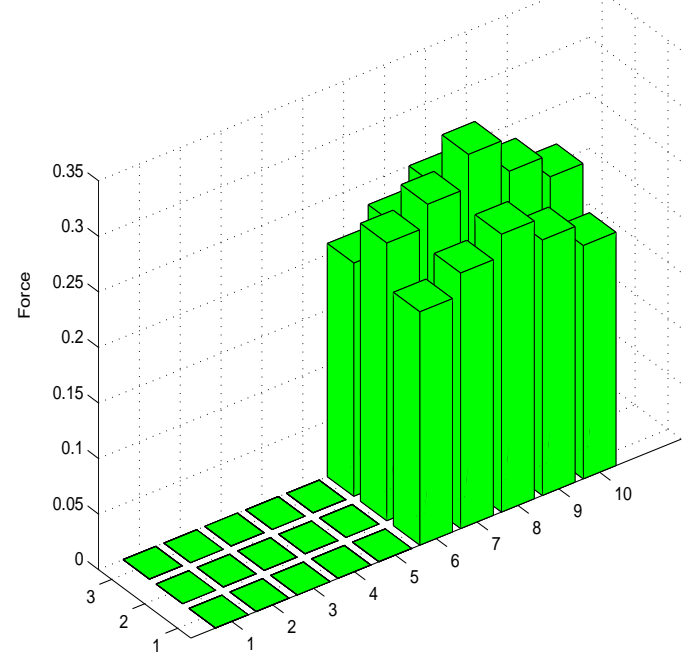

Fig. 10. An example plot of the tactile information

In addition a future design will possibly be 4-by-8 instead of 3-by-10, to provide the surgeon with a broader sensing area.

As far as the display is concerned, we will also include optical position sensors to the motors if necessary. In this way we can count each motor revolution and provide position feedback. The final step will be to do a comprehensive psychophysical evaluation of the display.

\section{ACKNOWLEDGMENTS}

The research is funded by the Research Council of Norway, grant 147830/320. The authors would like to thank Dr. Ronald Mårvik at St.Olavs Hospital for useful discussions and inputs related to Minimally Invasive Surgery. We would also like to acknowledge the engineering workshop at the Department of Engineering Cybernetics for help with mechanical and electronic designs and Eivind Sexe for testing on the micro motors.

\section{REFERENCES}

[1] G. Murali Krishna and K. Rajanna, "Tactile Sensor Based on Piezoelectric Resonance", Proceedings of the First IEEE International Conference on Sensors, vol. 2, pp.1643-1647, 2002.

[2] R.D. Howe, "Introduction to Haptic Display: Tactile display", Harvard University, http://haptic.mech.nwu.edu/intro/tactile/, unpublished.

[3] W.J. Peine, Remote Palpation Instruments for Minimally Invasive Surgery, Ph.D Thesis, Harvard University, 1998.

[4] D.T.V. Pawluk, J.S. Son, P.S. Wellman, W.J. Peine and R.D. Howe, "A Distributed Pressure Sensor for Biomechanical Measurements", Journal of Biomechanical Engineering, vol. 102, pp. 302-305, 1998.

[5] P. Pagh, W.B. Heginbothan and C.J. Page, "Novel Techniques for Tactile Sensing in a Three Dimensional Environment", Ind. Robot., pp.35-48, 1978.

[6] S. Sugiyama, K.K.M. Yonendu and I. Igarashi, "Tactile Image Detection Using 1K-Element Silicon Pressure Sensor Array", Sensors and Actuators, pp.397-400, 1990. 
[7] K. Tanie, "Advances in Tactile Sensors for Robotics", Proceedings of 6th Sensor Symposium, pp.63-68, 1986.

[8] G. Moy, C. Wagner and R.S. Fearing, "A Compliant Tactile Display for Teletaction", IEEE Int. Conference on Robotics and Automation, 2000

[9] D.T.V. Pawluk, C.P. van Buskirk, J.H. Killebrew, S.S. Hsiao and K.O. Johnson, "Control and Pattern Specification for a High Density Tactile Array", IMECE Proceedings of the ASME Dyn. Sys. and Control Div., vol. 64, pp.97-102, 1998.

[10] V. Hayward and J.M. Cruz-Hernández, "Tactile Display Device Using Distributed Lateral Skin Stretch", Proceedings of the ASME, vol. DSC-69-2, pp.1309-1314, 2000.

[11] C.R. Wagner, S.J. Lederman and R.D. Howe, "A Tactile Shape Display Using RC Servomotors", Proceedings of the 10th Symposium on Haptic Interfaces for Virtual Environment and Teleoperator Systems, pp.354-356, 2002.

[12] A.C. Guyton amd J.E. Hall, Textbook of Medical Physiology, Tenth Edition, W.B. Saunders Company, 2000.

[13] J.M. Lee, C.R. Wagner, S.J. Lederman and R.D. Howe, "Spatial Low Pass Filters for Pin Actuated Tactile Displays", Proceedings of the 11th Symposium on Haptic Interfaces for Virtual Environment and Teleoperator Systems, pp.57-62, 2003. 